\title{
Narrativa
}

\section{La costruzione della frontiera. La rappresentazione di Napoli in Braucci, Parrella e Pascale}

\section{Matteo Moca}

\section{(2) OpenEdition}

Journals

Edizione digitale

URL: https://journals.openedition.org/narrativa/697

DOI: 10.4000/narrativa.697

ISSN: 2804-1224

\section{Editore}

Presses universitaires de Paris Nanterre

\section{Edizione cartacea}

Data di pubblicazione: 1 décembre 2017

Paginazione: 129-138

ISBN: 978-2-84016-289-6

ISSN: 1166-3243

Notizia bibliografica digitale

Matteo Moca, «La costruzione della frontiera. La rappresentazione di Napoli in Braucci, Parrella e Pascale», Narrativa [Online], 39 | 2017, online dal 01 décembre 2021, consultato il 14 janvier 2022. URL: http://journals.openedition.org/narrativa/697 ; DOI: https://doi.org/10.4000/narrativa.697

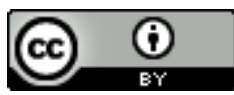

Narrativa est mise à disposition selon les termes de la Licence Creative Commons Attribution 4.0 International. 


\section{La costruzione della frontiera. La rappresentazione di Napoli in Braucci, Parrella e Pascale}

\section{Frontiera/Confine/Soglia}

Il concetto di frontiera attraversa la storia dell'uomo sin dalla sua origine, acquisendo un ruolo decisivo per il processo di auto-definizione dello stesso, con la consapevolezza che oltre una certa distanza, oltre un certo limite, ci sono cose diverse, differenti. Luoghi invisibili ad occhio nudo, ma pure sempre presenti nelle coscienze. Si tratta altresì di uno strumento che permette di fuggire l'illimitato, distinguendo, ad esempio, ciò che è abitato da ciò che non lo è:

Occupare uno spazio significa distinguere ciò che è abitato da ciò che non lo è, [...] fondando l'ordine a partire dal caos. In altre parole abitare non significa solo creare luoghi, ma anche non-luoghi, spazi altri. Il delimitare - atto di fondazione del luogo e dunque dell'abitare - implica l'istituzione di una dualità, qualunque essa sia - interno/esterno, ordine/disordine, limitato/illimitato, luogo/spazio, identità/alterità - ovvero significa concepire l'esistenza non solo del sé ma anche di qualcosa di altro-da-sé, un qualcosa certamente più incerto, sfumato, indeterminato, difficilmente qualificabile, ma altrettanto "reale"1.

I concetti di limite, confine e frontiera perdono in italiano la distinzione semantica che invece li contraddistingue in altre lingue ${ }^{2}$, ma resta il fatto che oggi, in un mondo globalizzato, queste definizioni, assumendole in senso più

1. Natali, Fabio, L'ambigua natura della frontiera. Antropologia di uno spazio teræo, Urbino, Quattroventi, 2007, p. 51.

2. Si pensi ad esempio alla distinzione semantica della lingua inglese dove i vari termini che definiscono confine, limite e frontiera (border, boundary, frontier) mantengono una loro specificità. Si veda su questo Bertazzoli, Raffaella, "In margine", in Monti, Silvia, Bellomi, Paola (a cura di), Froniere, confini, limiti, soglie. Fronteras, limites, confine, umbrales. Boundaries, Limits, Boders, Tresholds, Verona, Edizioni Fiorini, 2013, pp. V-VI. 
generale, hanno cambiato non poco i loro connotati, sono state sfumate le loro rigidità trasformando questi in concetti più fluidi e quindi in luoghi non solo di fermata obbligata, ma anche di attraversamento ${ }^{3}$. Ciò che comunque accomuna queste parole, seppure con le sfumature differenti ricordate, è la loro appartenenza ai campi semantici opposti di assimilazione e contrapposizione. Strettamente collegato a questi termini è quello di soglia, che rappresenta la sperimentazione del confine, del limite come passaggio o, al contrario, come spazio ultimo in cui fermarsi prima dell'oltrepassamento ${ }^{4}$. Si tratta, anche in questo caso, di un concetto fondamentale per comprendere appieno la natura dell'uomo contemporaneo, come nota Franco Rella quando scrive: "Se dovessi oggi indicare la figura del moderno, indicherei la figura della soglia: della frontiera che si fa fluida e sfrangiata; che si fa transito". La frontiera dunque, e tutti i concetti ad essa collegati, sono oggi, come definito da Alessandro Leogrande, il "termometro del mondo, qualcosa che si dilata nello spazio e nel tempo". Nel nostro tempo attuale dunque, dove le tensioni geopolitiche costituiscono le maggiori forze in campo ed è in atto un'omologazione del pianeta che sfuma sempre più confini e limiti, è lecito sottoporre ad esame le motivazioni che portano alcuni scrittori napoletani come Maurizio Braucci, Antonio Pascale e Valeria Parrella, autori dai tratti assai netti e riconoscibili, in una direzione contraria, concentrando la propria narrazione non solo nel proprio Sud, ma nell'ambiente più ristretto della città o addirittura di una sua parte. Tale aspetto diviene senza dubbio un luogo di interesse critico nella discussione sui confini e le frontiere proprio perché è in netta controtendenza con l'andamento della società contemporanea. La scelta di questi autori, che come si vedrà scrivono in un arco di

3. Resta comunque indubbio il fatto che, in alcune parti del mondo, le frontiere hanno mantenuto o ancor di più rinforzato il loro ruolo di fermata. Basti pensare alle frontiere di luoghi che danno accesso alle rotte del Mediterraneo, oppure ai tentativi di costruzione di frontiere invalicabili anche nel cuore dell'Europa.

4. Si pensi ad esempio alla soglia del Purgatorio descritta da Dante nel canto IX del Purgatorio della Divina Commedia; esempio perfettamente calzante perché, nella sua descrizione, Dante riunisce i caratteri di fermata e attraversamento: "Noi ci appressammo, ed eravamo in parte / che là dove pareami prima rotto, / pur come un fesso che muro diparte, / vidi una porta, e tre gradi di sotto / per gire ad essa, di color diversi, / e un portier ch'ancor non facea motto. / E come l'occhio più e più v'apersi, / vidil seder sovra 'l grado sovrano, / tal ne la faccia ch'io non lo soffersi; / e una spada nuda avëa in mano, / che reflettëa i raggi sì ver' noi, / ch'io drizzava spesso il viso in vano." (Alighieri, Dante, La Divina Commedia, Vol. II Il Purgatorio, Firenze, La Nuova Italia, 1977, pp. 99-100).

5. Rella, Franco, Miti e figure del moderno, Milano, Feltrinelli, 2003, p. 15.

6. Leogrande, Alessandro, La frontiera, Milano, Feltrinelli, 2015, p. 37. 
tempo molto ravvicinato, deriva quindi in primo luogo dal loro comune rifugio nella scrittura, quello che non si muove dai propri luoghi, ma anche da un aspetto stilistico e narrativo che, come si tenterà di evidenziare, porta ad un naturale raggruppamento. La soglia assume allora un valore spaziale che oltrepassa il semplice significato di zona di passaggio o confine, diviene spazio materiale, zona grigia abitabile in cui dentro e fuori divengono indistinguibili, "La soglia [Schwelle] è una zona"’.

\section{Il SOTTOSUOLO MERIDIONALE}

Per procedere in questa analisi, e per valutare anche la categoria di Sud, è interessante partire da più lontano ${ }^{8}$, ovvero da uno scritto di Ignazio Silone emblematicamente intitolato La narrativa e il "sottosuolo meridionale", apparso la prima volta nel 1956 sulla rivista Quaderni di prospettive meridionali. In apertura dello scritto, Silone definisce subito con nitidezza la sua posizione:

In confronto ad altri modi, questo criterio storico-geografico-sociale della cosiddetta meridionalità non è proprio, mi sembra, il più ozioso e sterile. Purché, si intende, anche con esso non si abusi: purché non si dimentichi che le comuni caratteristiche di tale "meridionalità" si ritrovano poi, nelle singole opere, in misura assai variabile?.

Seppur la premessa di Silone possa apparire oggi banale, è altresì indubbio come spesso si tenti di incasellare autori ed opere in categorie che non ne rispecchiano la portata. Silone in ogni caso rintraccia nella scrittura di autori meridionali delle peculiarità, indagando la marginalità economica del Sud d'Italia rispetto ad un modello di progresso di matrice nordica che, ancora oggi, segna una differenza molto grande:

7. Benjamin, Walter, I Passages di Parigi, Torino, Einaudi, 2000, p. 555.

8. Tale operazione è legittimata anche dal rapporto che gli scrittori di questa generazione intrattengono con la letteratura. È infatti indubbio come i riferimenti letterari siano, soprattutto, autori moderni o addirittura contemporanei, come Anna Maria Ortese (in particolar modo decisiva nell'opera di Valeria Parrella) e Raffaele La Capria. Su questo particolare passaggio, si vedano i capitoli "Napoli e Caserta", in LA PORTA, Filippo, Uno sguardo sulla città. Gli scrittori contemporanei e i loro luoghi, Roma, Donzelli, 2010, pp. 53-60, oppure l'intervista a Parrella, SERri, Mirella, "Con il pilota di Liala il primo volo su Napoli”, in La Stampa, 29 dicembre 2007.

9. Silone, Ignazio, Romanzi e saggi, Vol. II, Milano, Mondadori, 1999, p. 1396. 
Il Sud non è solo una nozione geografica ma anche storica e sociale, in antagonismo con il Nord. Quasi ovunque il Sud è più povero del Nord; quasi ovunque è prevalentemente agricolo, mentre il Nord è industrializzato. Sarebbe una stravaganza voler calcare la mano su queste e altre analogie storico-naturali per estrarne una qualunque teoria; ma nulla ci vieta di apprezzare convenientemente il fatto che un racconto siciliano o abruzzese possa essere accolto nella Virginia, in Ucraina, in Indonesia come la narrazione di una vicenda locale ${ }^{10}$.

Il Sud nello stesso tempo deve essere considerato, sempre secondo Silone, come un insieme di varietà regionali con caratteri precisi e specifiche differenze, integrandole in un sistema di relazioni più vaste, dove però solo la reale conoscenza delle ragioni profonde che hanno portato alla nascita di alcuni tratti peculiari, di certi stereotipi o, come li chiama Silone, "apparenze", può generare una narrativa che parta da intenti dichiaratamente realistici non restando solamente descrittiva. $\grave{E}$ questa una caratteristica che unisce i testi in questione, in particolar modo quelli di Braucci e Pascale, dove la narrazione si fa molto realistica tanto da confondere le sue linee con quelle del reportage ${ }^{11}$.

\section{Parrella, Braucci e Pascale: NAPOLI E CASERTA}

Le opere dei tre autori in questione afferiscono alla loro prima produzione e riguardano un arco di tempo assai ristretto che va dal 1997 al $2003^{12}$. Questi anni non sono stati casuali per la letteratura che aveva come tema il Sud e coinvolgeva scrittori meridionali: sono gli anni in cui emergeva con grande forza una originalità letteraria, con opere singole o raccolte di racconti, prima che le leggi del mercato editoriale inglobassero la naturalezza di questa scrittura per replicarla su ampia scala ${ }^{13}$. Ciò su cui in ogni caso tenteremo di interrogarci,

10. Ibid., p. 1371.

11. Si pensi ad esempio al libro di Roberto Saviano, Gomorra, emblematico circa il rapporto tra narrativa romanzesca e reportage di cui si parla.

12. Solo la raccolta di Valeria Parrella Mosca più balena e la raccolta collettanea Pensa alla salute sono del 2003; le opere di Braucci e Pascale qui analizzate invece risalgono agli anni tra il 1997 e il 1999.

13. Il punto di rottura, che scatenò infatti molte discussioni sulla stampa, fu l'uscita della raccolta edita da Einaudi Disertori. Sud: racconti dalla frontiera nel 2000, dove figurano, tra gli altri, anche Pascale e Braucci. Prima di questa raccolta, ce ne furono altre, pubblicate da più piccoli editori, $\mathrm{ma}$ forse più genuine ed originali. Si fa qui riferimento in particolare a FOFI, Goffredo (a cura di), Narrare il Sud. Percorsi di scrittura e di lettura, Napoli, Liguori, 1995; ID. (a cura di), Luna nuova. Nuovi scrittori dal Sud, Lecce, Argo, 
attraverso le opere di cui parleremo, sono innanzitutto le motivazioni che portano questi autori a sfuggire la frontiera e addirittura a chiudere le loro narrazioni nella città conosciuta oppure, come è il caso di Braucci, in un solo quartiere. Nella prefazione alla raccolta Luna nuova, il curatore Goffredo Fofi scrive:

La prima ricchezza dell'Italia sta nella diversità delle sue origini e delle sue città, stava nella varietà delle sue tradizioni e culture. Contro di essa si è accanita e si va ancora accanendo la storia, e un'idea di sviluppo, incurante di ogni differenziazione e libertà, di ogni ricerca esperimentazione ${ }^{14}$.

Con questa riflessione Fofi sembra proseguire il discorso cominciato decenni prima da Silone, a cui infatti, nella prefazione, fa riferimento. In questa raccolta le ambientazioni sono limitate, come detto, ai luoghi familiari agli scrittori, ma questo indica anche contraddizioni forti, difficoltà grandi nell'adattarsi ad un contesto dove la rivolta verso l'esterno è complicata e solo la delinquenza e lo sberleffo portano alla conquista di nuovi spazi e luoghi. Resta comunque intatta la teorizzazione di Silone: gli autori parlano di un ambiente che è loro familiare e di cui conoscono bene gli elementi.

Nel racconto di Pascale La controra, per esempio, viene messo in scena un momento significativo della giornata, la controra del titolo, appunto, ovvero quelle ore durante le quali non è possibile uscire di casa poiché il caldo è così penetrante che porta alla malattia:

D'estate scendevo in giardino alle cinque di pomeriggio. Prima non si poteva. Me lo vietava mio padre: "Deve passare la controra", o anche "Durante la controra si deve dormire". Perché la controra era un limite: superarlo significava entrare in un territorio assolato - e rischioso - che non potevo permettermi di abitare (e di immaginare $)^{15}$.

Il momento della giornata diventa allora un limite problematico da superare, una zona di confine che, come scrive Pietro Zanini nel suo saggio Significati del confine, "indica un limite comune, una separazione tra spazi contigui; è anche un modo per stabilire in via pacifica il diritto di proprietà di ognuno in un territorio

1997 e Cappeldi, Gaetano, Trecca, Michele, Verrengia, Enzo (a cura di), Sporco al sole: racconti del sud estremo. Prima antologia dei narratori meridionali under 25 o quasi, Lecce, Besa, 1998.

14. Fofi, Goffredo (a cura di), Luna nuova. Nuovi scrittori dal Sud, cit., p. 7.

15. Pascale, Antonio, La controra, in Fofi, Goffredo, Luna nuova. Nuovi scrittori dal Sud, cit., p. 116. 
conteso"16. Questo territorio liminale diviene luogo di contesa quando il padre e la madre del protagonista bambino iniziano a discutere frequentemente, tanto da costringere il figlio a disobbedire ai loro comandi, a mandarlo fuori di casa durante l'ora letale, quella dove i raggi del sole e i relativi effetti provocano danni all'organismo. Questo porterà a una trasformazione del corpo nel figlio, che diverrà, da agile e scattante, a sovrappeso e ingolfato, ferito dal clima della città:

Come per osmosi il calore che mi aveva preso e invaso quella notte, avrebbe richiamato di giorno altro calore: sarei ingrassato; il seno si sarebbe gonfiato, poi il ventre avrebbe formato strati su strati fino a coprirmi l'inguine, e infine il collo, gonfio e molle, avrebbe infossato il mento e con esso la testa parve scivolare tra le sabbie mobili del tronco ${ }^{17}$.

La stessa ferita del corpo ritorna in un altro racconto di Pascale, intitolato Il mare magnum delle possibilità e raccolto in Pensa alla salute, dove l'esperienza dell'infortunio, causato stavolta da una bravata con il motorino, torna a pesare sul corpo. "La nostra pelle è la nostra storia, non possiamo cancellare le rughe"18, dice il padre al figlio all'ospedale di Napoli. Non si tratta di un male dissimile da quello che fa la sua comparsa in La controra: il male è una condizione che non può essere esclusa dalla città e con cui, volontariamente, ma più spesso involontariamente, i protagonisti di questi racconti devono fare i conti.

Nella stessa raccolta figura anche il racconto di Valeria Parrella La tasca davanti, dove l'ambientazione napoletana, e in senso più generale meridionale, costituisce il fondamento su cui è edificato il racconto: si tratta di un caso di malasanità considerato in opposizione rispetto alla stessa situazione in un ospedale di Milano, città nella quale l'amica della protagonista si è trasferita. A Napoli non ci sono i soldi per i corsi pre-parto, che quindi vengono fatti da volenterosi medici e infermieri senza che l'ospedale lo sappia. A questi partecipa la protagonista che, per non sfigurare nei confronti dell'amica ormai residente a Milano, nei suoi racconti inventa l'esistenza di una piscina all'interno dell'ospedale, utile per il rilassamento successivo agli esercizi di preparazione al parto. Tale però diventa la forza delle donne che seguono questi segreti corsi pre-parto, che i contorni della realtà si fanno più sottili e anche la donna milanese, tornata a

16. Zanini, Pietro, I significati del confine. I limiti naturali, storici, mentali, Milano, Bruno Mondadori, p. 10.

17. Ibid., p. 114.

18. Pascale, Antonio, Il mare magnum delle possibilità, in Aa.Vv., Pensa alla salute, Napoli, l'ancora del mediterraneo, 2003, p. 59. 
Napoli per il parto dell'amica, crede all'esistenza della piscina. In contrasto verso una situazione complessa e difficile, i rapporti personali si fanno molto forti e i legami inscindibili:

Si guardavano, ma i loro occhi andavano oltre la curiosità: le donne assorbivano il loro dolore, come un branco. Come compagne di scuola a un esame, impedivano che la sua ansia diventasse solitudine. Appena arrivava una contrazione respiravano per sincrono vitale tutte assieme ${ }^{19}$.

Anche la raccolta di racconti di Parrella Mosca più Balena vive di un'ambientazione esclusivamente napoletana, dove la narrazione si affida a un tessuto minuto e poco appariscente che riesce però a tratteggiare con forza l'immagine di una esistenza napoletana qualunque, affogata nella vita dei quartieri, nelle questioni quotidiane e con protagoniste donne delle più diverse estrazioni sociali: si incontrano così la donna che tenta di fronteggiare la criminalità dilagante, chi si reca regolarmente a sostenere concorsi statali tra mille difficoltà o una piccola bambina che salva la sua famiglia da un terremoto.

Il racconto Dritto dritto negli occhi, per esempio, ricostruisce la storia di una donna che aspira al benessere e si serve della camorra e dei suoi esponenti per raggiungerlo, finché non raggiunge la consapevolezza che per arricchirsi veramente deve trovare un uomo di più alto rango: pensa così che l'uomo giusto sia un parlamentare, che riesce a sposare. In questo racconto esiste una differenza sostanziale con La tasca davanti perché, laddove quest'ultimo era la narrazione di una lotta contro la realtà, qui invece si tratta di una rassegnazione e del tentativo di dominare il mondo criminale in cui la protagonista si è trovata a vivere, senza avere minimamente in mente la possibilità di un cambiamento. Ecco perché (cosa rara che non succede quasi mai nei racconti e romanzi di cui si sta parlando), la donna lascerà Napoli per andare a Roma in compagnia del nuovo marito, lasciando così la terra partenopea: non è possibile definire se si tratti di una sconfitta o di una vittoria, ciò che è certo, invece, è che si tratta di un superamento del limite, della frontiera, proprio perché ci si è accontentati di quel mondo che quindi, dopo averne avvertito le strettezze e le forzature e dopo aver approfittato di queste, costringe a partire.

In questa raccolta Parrella racconta, commenta, riflette e ferma la realtà che le scorre davanti per ritrovarla nell'angolo in cui la luce della riflessione riesce a rischiararne, anche solo per un momento, la natura. Per esempio, in Asteco e cielo,

19. Parrella, Valeria, La tasca davanti, in Aa.Vv., Pensa alla salute, cit., p. 52. 
altro racconto della raccolta, così descrive il paradosso urbanistico del palazzo delle Vele di Scampia in cui abita:

Il mio quartiere ha il numero di una legge, 167 . Un giorno prendo un codice e vedo cosa significa. A volte gli studenti di architettura salgono sull'autobus e si fanno il giro tra i palazzi, scattando le foto dai finestrini come i giapponesi, non si azzardano a scendere. Li vedi a bocca aperta incantati dalle forme e dalla fantasia. In una di queste fantasia ci vivo io. L 48167 LOTTO 11 ISOLATO 20 SCALA A PIANO 9 ASCENSORE ROTTO20.

In sintonia con altri scrittori napoletani dunque, i dettagli vengono colti nel loro potere evocativo di alludere a una condizione sociale e antropologica, propria della letteratura meridionale: ne emerge dunque una mappatura precisa del quartiere e, di conseguenza, di Napoli, che viene qui rappresentata divisa in fasce sociali ben precise che appartengono e abitano aree urbanistiche chiuse.

Mascherato da romanzo, ma procede in realtà per successione di brevi racconti, è Il mare guasto di Maurizio Braucci, che intrattiene una certa vicinanza con l'ambientazione di Mosca più Balena. Il libro di Braucci è addirittura ambientato tutto in un unico quartiere napoletano, il quartiere Santo, dove l'autore individua un microcosmo autosufficiente in cui si muovono, in maniera armoniosa e veloce, gli stessi personaggi, tutti spinti dall'unico ordine conosciuto, che è quello della camorra e dei suoi ritmi che governano il quartiere. Ne viene fuori un dramma corale, quasi verghiano ${ }^{21}$, in cui comanda un codice illegale ma comune a tutti. La camorra è qui colta non tanto nelle sue manifestazioni più eclatanti quanto nella sua più subdola e capillare penetrazione nel quotidiano e nell'immaginario di ogni abitante, come perfettamente mostrato dall'intreccio dei personaggi che, volenti o nolenti, sono costretti ad incrociare nella loro vita la macchina criminale. Nasce così un cosmo chiuso in se stesso, che non sogna neanche l'uscita dal quartiere, perché solo lì si rintraccia tutto l'orizzonte di esplorazione. Questo procedimento di specificazione assoluta dell'ambientazione è ben chiaro già dall'incipit del romanzo che, partendo proprio da una descrizione del cosmo, finisce nel piccolo quartiere napoletano:

È la stessa storia, che questo pianeta è una palla di metallo sospesa di fronte al sole e che si arroventa per diventare un sole pure lei in un batter di ciglia, in

20. Parrella, Valeria, Asteco e cielo in Ead., Mosca + Balena, Roma, minimum fax, 200, p. 112.

21. Per questa interessante comparazione, si veda Carmosino, Daniela, Uccidiamo la luna a Marechiaro. Il sud nella nuova narrativa italiana, Roma, Donzelli, 2009. 
un istante, e che tutti noi evaporiamo e poi ci condensiamo finché, un giorno, non avremo più un posto dove tornare e ci disperderemo nell'universo, come fumo tra nebulose. [...] Così, quel giorno, nel quartiere Santo stava parlando l'aria della primavera, tante persone per la strada erano in affari con la vita e uno, Rodrigo, un grosso giovane dalla pelle bruna, se ne stava seduto in una 127 obsoleta in attesa dei clienti per la sua cocaina di mediocre qualità ${ }^{22}$.

Spostandosi da Napoli a Caserta, si incontra infine il romanzo-saggio a carattere sociologico La città distratta di Pascale, edito per la prima volta nel $1999^{23}$. L'ambientazione esclusivamente casertana è composta di voci, odori e colori, tutti segnali che l'autore tenta di decifrare, con l'obiettivo di costruire un ritratto fedele e preciso. L'occhio di Pascale assume allora un carattere investigativo, tarato sulla giusta distanza dalle cose, che unisce la precisione di un antropologo con una emotività mai stucchevole proprio perché sincera. Il testo di Pascale, però, a differenza delle altre opere qui analizzate, compie un importante salto in avanti: la città casertana, con tutte le sue peculiarità, i giovani, gli extra-comunitari, il culto di Padre Pio, parla in realtà di tutta l'Italia, non fermandosi ai soli confini della città. Si intuisce già dalle prime pagine del libro, quelle in cui Pascale riporta:

Il fatto è che Caserta è piena di ex (gli ex casertani sono soliti cambiare le facce e non i vestiti. L'assessore alla cultura veste ancora come quando era di estrema sinistra, si presenta sciatta e trasandata). Ci sono ex maoisti, ex democristiani, ex contadini, ex comunisti, ex socialisti: non ci sono, invece, ex fascisti. Ci sono solo fascisti. Ma non mancano ex preti, ex imprenditori, ex negozianti, ex impresari, ex atleti, ex sportivi. E quelli che non sono ancora ex sembrano sempre in procinto di diventarlo ${ }^{24}$.

La descrizione minuziosa delle trasformazioni sociali e culturali della città va oltre la semplice inchiesta e, in maniera subliminale, oltrepassa la frontiera meridionale e dipinge un quadro tanto complesso quanto fedele. Questo caso di Pascale è, come detto, l'unica testimonianza di una fuoriuscita, ma anch'essa però vive di un radicamento meridionale anche se suggerisce un'importante chiave di lettura, quella di poter leggere la società e la cultura italiane attraverso

22. Braucci, Maurizio, Il mare guasto, Roma, Edizioni E/O, 2010, p. 11.

23. La prima edizione del volume è apparsa appunto nel 1999 per la casa editrice l'ancora del mediterraneo; la versione da cui si citerà è invece quella Einaudi, uscita nel 2001.

24. Pascale, Antonio, La città distratta, Torino, Einaudi, 2001, pp. 5-6. 
un filtro meridionale che si fa universale, mettendo quindi in dubbio l'esistenza di una netta separazione.

\section{Conclusione}

Agli interrogativi che si ponevano in apertura del saggio, è forse possibile a questo punto tentare una sfuggevole risposta, elaborata a partire da questi mezzi. Le opere di Parrella, Braucci e Pascale procedono tutte per giustapposizione di dettagli, come se solo l'insieme di essi possa dare un quadro esaustivo, attraverso dei fermi-immagine fondamentali per leggere la città di Napoli e dintorni, perché da lì mai ci si è allontanati. Le motivazioni di questa scelta non possono certo essere espresse con certezza assoluta, ma è indubbio che nei moderni tempi di dilatazione della frontiera che via via si allarga e si fa sempre più liquida, questa scrittura, in modi ovviamente diversi, resti chiusa, bloccata, dai confini della città che non vengono mai oltrepassati. La decisione può ovviamente avere più motivi, ma forse il più notevole è quello di una resistenza forte allo scorrere delle cose e dei tempi, ed è il fatto che la terra da cui si proviene assume un ruolo precipuo, per la ricchezza delle tradizioni e per i modi di vivere, che porta a muoversi al suo interno cercando una comprensione $o$, tentativo più complesso e raro, un cambiamento.

Matteo Moca

Università di Bologna e université Paris Nanterre (CRIX-EA 369 Études Romanes) 